\title{
Using I-TOF spectrometry to measure photon energies at FELs
}

\author{
P.N. Juranić,,$^{a, 1}$ M. Martins, ${ }^{b}$ J. Viefhaus, ${ }^{a}$ S. Bonfigt, ${ }^{a}$ L. Jahn, ${ }^{b}$ M. Ilchen, ${ }^{a}$ \\ S. Klumpp ${ }^{b}$ and K. Tiedtke ${ }^{a}$ \\ ${ }^{a}$ Deutsches Elektron-Synchrotron (DESY), \\ Hamburg, Germany \\ ${ }^{b}$ University of Hamburg, \\ Hamburg, Germany \\ E-mail: pavle.juranic@desy.de
}

\begin{abstract}
Due to the stochastic nature of the Self Amplifying Spontaneous Emission (SASE) process and the resulting pulse-to-pulse fluctuations of the Free Electron Laser (FEL) photon energies, experimenters working with FELs need to get real-time feedback about the photon properties for their experiments. Investigations of narrow atomic or molecular resonances, phase transitions, or any other kind of effect heavily dependent on photon energy would need to know the precise FEL photon energy for each individual photon bunch. Furthermore, any spectrometer developed to deliver the information of these properties should not significantly interfere or degrade the FEL beam. Therefore, the group at the Free Electron Laser in Hamburg (FLASH) has developed an online photoionization spectrometer that uses ion time of flight (I-TOF) measurement methods on noble gases to measure the photon energy of each pulse. This paper presents the first test results for the viability of this online photoionization spectrometer (OPS).
\end{abstract}

KEYWORDS: Gaseous detectors; Instrumentation for FEL; Ionization and excitation processes; Beam-line instrumentation (beam position and profile monitors; beam-intensity monitors; bunch length monitors)

\footnotetext{
${ }^{1}$ Corresponding author.
} 


\section{Contents}

1 Introduction 1

2 The I-TOF spectrometer 2

$\begin{array}{lll}2.1 & \text { Theory } & 2\end{array}$

2.2 Description of the device $\quad 4$

3 Experiment 5

3.1 The beamline $\quad 5$

3.2 Experimental procedures and parameters 5

4 Data analysis 6

$\begin{array}{lll}5 & \text { Results and discussion } & 7\end{array}$

$\begin{array}{lll}6 & \text { Conclusions } & 7\end{array}$

\section{Introduction}

The accurate selection of photon energies at experiments at synchrotron facilities is largely a nonissue due to the monochromators and variable gap undulators used to change the photon energy of the beam delivered to most beamlines. Once calibrated, they deliver the same photon energy at the exit slit for a certain monochromator angle and undulator gap size. However, the Free Electron Laser in Hamburg (FLASH) uses fixed gap undulators [1] to produce super-brilliant vacuum ultraviolet (VUV) light via a self amplifying spontaneous emission (SASE) process [2, 3]. To get the high flux beam at a fixed photon energy, the electron beam energy is the only variable parameter [2]. The tuning of the photon energy is not easy compared to a monochromator-undulator combination found at a classic synchrotron. Similarly, because the electron bunch settings need to be reset for every new photon energy and various effects constantly compensated due to the stochastic nature of the SASE process, it is not possible to always determine the exact photon energy of the outcoming light. The standard process in setting the FEL radiation to a certain photon energy involves the adjustment of the electron beam properties while a mirror deflects the FEL beam onto a grating spectrometer [2]. However, the energy of the electron beam that produces the photons fluctuates enough to cause the photon energy to fluctuate within the FEL's bandpass, which is about $1 \%$ at FLASH, and may also cause the photon energy to drift over time by as much as 1 to $2 \%$ if not carefully monitored. Since keeping a mirror inside the beam to constantly monitor the photon energy is not an option if one wants to also use the beam for experiments downstream, a new method needed to be developed that would give the operators information about the photon energy of the FEL beam without significantly affecting the beam. FEL users would also greatly benefit from the 
development of this new monitoring method, being able to record the photon energies of each FEL pulse as they are taking data. When completed, this new method would work in tandem with the already existing beam-position monitors (BPMs) and light-intensity measuring gas monitor detectors (GMDs) [4] to give the users and operators complete knowledge of the photon pulses at an FEL.

The photon diagnostic group at FLASH has come up with several designs to answer this challenge. One such design is the variable-line spacing (VLS) grating spectrometer [4]. However, the VLS grating spectrometer has limitations when used in the higher end of the soft X-ray region, and is not suited for the hard X-ray region that would be reached by future FELs. Another design uses electron time of flight (e-TOF) spectrometry to measure the photon energies of the FEL beam [5]. While this method could work well at almost all photon energies, the influence of magnetic fields, which are inevitable close to the accelerator structure, and the small fraction of the full solid angle, which is typically detected, may lead to a relatively low count rate and correspondingly higher uncertainties. In order to circumvent those two problems, the photon diagnostic group at FLASH has developed an ion time of flight (I-TOF) spectrometer to measure the photon energies of individual FEL pulses by using the relations between the partial photoionization cross sections of noble gases.

\section{The I-TOF spectrometer}

\subsection{Theory}

Over the course of the last century, many experiments have been performed to find the singlephoton photoionization cross sections of various substances. The experiments that measured both the absolute and partial photoionization cross sections of noble gases were among the simplest and easiest to perform, yielding a large number of accurate cross section measurements for gases like xenon, argon, neon, and krypton in the soft x-ray region. A good compilation of the results from the above-mentioned references can be found in the review of Tiedtke et al., along with the references to articles with the cross section data [6]. Of the references listed in [6], of particular interest to this report are the neon data, found in [7-9]. The idea behind the I-TOF spectrometer was to use this large and accurate pool of data to evaluate the photon energies of FLASH. In addition there have also been theoretical calculations performed for the partial cross section of these gases, like for neon $[10,11]$.

The most important data for the application of the I-TOF spectrometer were the partial cross section measurements. When one measures the partial single-photon photoionization cross section of various charge states of a gas, the relative ratio between the charge states, like the single-todouble photoionization ratio, has a given value for a fixed photon energy. In general, these ratios change with a changing photon energy and can be used as a measure to determine the photon energy [6]. A commonly used value to express the relationship between the differently charged photoions is their ratio to the single photoionization partial cross-section, $\sigma^{n+} / \sigma^{+}$, where $\sigma^{n+}$ is the partial photoionization cross section for the ion with charge $n$. When this ratio is plotted against the energy of the ionizing photon, the curve for neon gives a unique value of the $\sigma^{2+} / \sigma^{+}$for every photon energy between the double photoionization threshold around $60 \mathrm{eV}$ and $200 \mathrm{eV}$, as seen in figure 3. With precise measurements, the spectrometer could use this uniqueness to measure the photon energy of the FEL beam. In context of measurements at a FEL, the photoionization ratios are only reliable as long as the photon flux is low enough so that only single-photon processes can occur. 


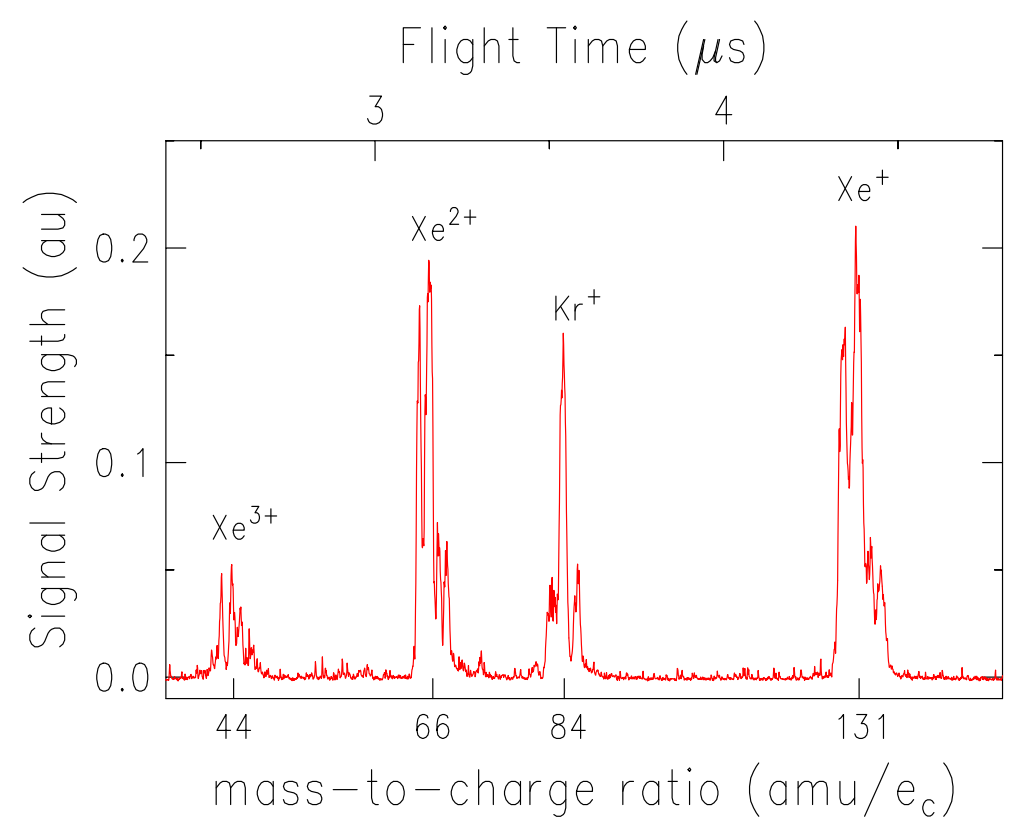

Figure 1. A typical I-TOF spectrum of a $\mathrm{Kr} / \mathrm{Xe}$ gas mixture taken at the BL2 beamline at FLASH at about $90 \mathrm{eV}$. The spiked peak structures are the isotopes of $\mathrm{Kr}$ and $\mathrm{Xe}$ which are clearly distinguishable due to the good resolution of the I-TOF spectrometer.

The peak brilliance of the FLASH FEL is about six orders of magnitude greater than the best third-generation synchrotron-radiation light sources can offer, and has a flux of about $10^{13}$ photons per pulse, with a duration of 10-50fs [3, 6], giving rise to worries that a lot of the processes the spectrometer may be detecting would be multi-photon ones. However, as the installation of the gas monitor detector at FLASH demonstrated, the photon-gas interaction in the unfocused FEL beam is in the single photon interaction regime [6]. Since the spectrometer would be best placed far out of the focus of the FEL beam to allow the experimenters to use the focus, the reference data obtained at a synchrotron radiation source will remain valid for the evaluation of the photon energy. To see whether the measurement statistics at a FEL would be sufficient for this kind of spectrometer, we took an I-TOF spectrum of a $\mathrm{Kr} / \mathrm{Xe}$ gas mixture during a brief commissioning beamtime at the BL2 beamline at FLASH. An average single-shot spectrum is shown in figure 1. The $\mathrm{Xe}^{2+}$ and $\mathrm{Xe}^{+}$peaks are clearly visible, and their ratio can be determined with an uncertainty smaller than $0.5 \%$. The uncertainty in the measurements is derived from the background noise to either side of the peaks.

In addition to being able to emit pulses with a single bunch of photons at a repetition of $5 \mathrm{~Hz}$, the FLASH FEL can also be used to emit pulses with multiple bunches in a single pulse train that can be set 1,2,10 or $100 \mu \mathrm{s}$ apart [4]. Further properties of FLASH are given in table 1, copied from [4]. The I-TOF spectrometer may not be able to measure the per-bunch photon energy for bunches with an interval of 1 or $2 \mu$ s apart, since the flight time of the ions could be smaller than the time between the photon bunches. However, it would even then be able to give an average photon energy for the whole pulse and all the bunches in it. Another concern is that the current from the ions on the multiplier may become too large due to a high gas pressure or photon flux and 
Table 1. Performance of FLASH.

\begin{tabular}{|c|c|}
\hline Parameter of FLASH & \\
\hline Wavelength range fundamental & $6.9-47 \mathrm{~nm}$ \\
\hline \multirow[t]{2}{*}{ Higher harmonics } & $3^{\text {rd }} \sim 2.3 \mathrm{~nm}$ \\
\hline & $5^{\text {th }} \sim 1.4 \mathrm{~nm}$ \\
\hline Pulse energy average & $10-50 \mu \mathrm{J}$ \\
\hline Peak power & several GW \\
\hline Pulse duration (FWHM) & $10-50 \mathrm{fs}$ \\
\hline Spectral width (FWHM) & $0.5-1 \%$ \\
\hline Spot size at the undulator exit (FWHM) & $\sim 160 \mu \mathrm{m}^{a}$ \\
\hline Angular divergence (FWHM) & $90 \pm 10 \mu \mathrm{rad}^{a}$ \\
\hline Peak brilliance & $10^{29}-10^{30}$ photons $/ \mathrm{sec} / \mathrm{mrad}^{2} / \mathrm{mm}^{2} / 0.1 \%$ bw \\
\hline
\end{tabular}

${ }^{a}$ SASE in saturation @30 nm

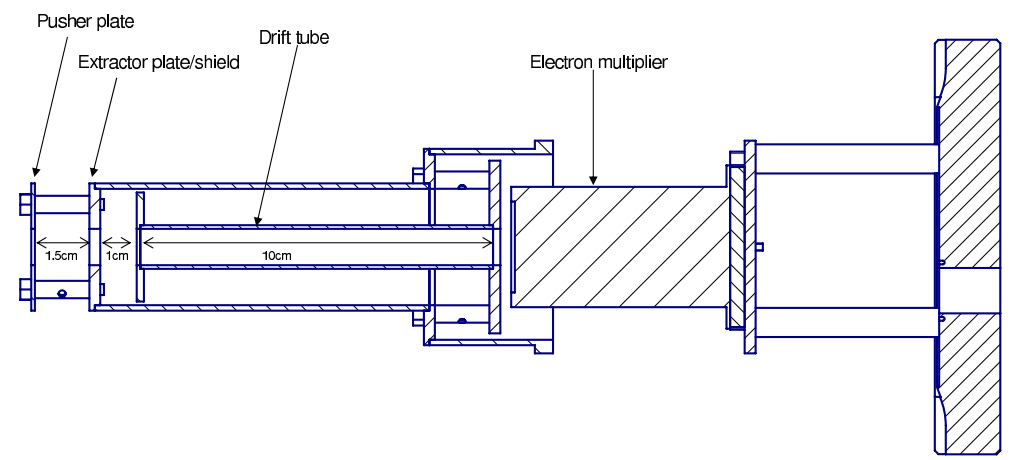

Figure 2. A schematic drawing of the I-TOF. The extractor plate is grounded and is a part of a cylinder that acts as a conductive shield for the drift tube and the electron multiplier. Immediately behind the drift tube is the electron multiplier. The whole apparatus is mounted on a CF63 flange. The interaction region lies between the pusher and extractor plate.

introduce non-linearities in the measurement of the spectra because of the output current limits for linear operation. However, this problem can be easily rectified by lowering the gas pressure in the interaction region or by lowering the multiplier gain until we are back in the linear regime for the multiplier. As an example, we expect to create about $10^{6}$ photoions in the interaction region per pulse for $\mathrm{Ne}$ at $90 \mathrm{eV}$ at a pressure of $10^{-6} \mathrm{mbar}$.

\subsection{Description of the device}

Ion time-of-flight spectrometers have been used for a long time, and the basic design for the high resolution I-TOF spectrometer has been laid out by Wiley and McLaren in 1955 [12]. Our I-TOF spectrometer design can be seen in figure 2 .

The ions are created in the interaction region between the pusher and extractor plates made of copper, and then pushed by a strong electric field towards the drift tube. We applied a potential of $1860 \mathrm{~V}$ to the pusher plate, while the extractor plate (which also serves as a shield) was kept 
grounded. The ions were sent towards the extractor plate, and were passing through a $1 \mathrm{~cm}$ diameter copper-mesh-covered hole towards the drift tube. A $-1900 \mathrm{~V}$ electrical potential was applied to the drift tube to time-focus the flight path of the photoions. At the end of the drift tube is a commercial 14880 series fast TOF electron multiplier from SGE, usually set to a potential of $-3000 \mathrm{~V}$. The drift tube, only $1 \mathrm{~cm}$ in diameter, is narrow enough to make sure that no stray ions will curve in towards the top of the electron multiplier and spoil the time resolution of the TOF.

\section{Experiment}

\subsection{The beamline}

The test of the accuracy and the calibration of the I-TOF spectrometer was carried out at the BW3 beamline at the DORIS III storage ring at the Deutsches Elektronen-Synchrotron (DESY) in Hamburg, Germany (next door to the FLASH FEL facility). We concentrated our measurements on photon energies between 20 and $200 \mathrm{eV}$, the energy range of the FLASH FEL at its present stage. To perform the calibration of the I-TOF spectrometer at the FEL directly would be impractical since the needed photon energy changes at the FEL are a complicated process that can take hours of tuning and would disrupt user operations.

The BW3 beamline at DORIS III has an energy range between 20 and $1500 \mathrm{eV}$, with the photon flux below $1000 \mathrm{eV}$ being on the order of $10^{12}$ photons $/ 0.1 \%$ bandwith [13-15]. Higher harmonics are present in the percent level below $50 \mathrm{eV}$, preventing the beamline's use below this energy for a straight forward calibration of the I-TOF. The beamline has stray light in this energy range. The resolution of the beam at the beamline had been set to less than $0.1 \%$ bandwith by suitable settings of the exit slit. This value has to be compared with the spectral width of the FLASH FEL, which is between $0.5 \%$ and $1 \%$ [4] at full width at half maximum (FWHM) of a pulse. Keeping in mind that one later wants to detect photon energy shifts within this width from shot to shot, the resolution at BW3 was sufficient to gauge the efficacy of the spectrometer.

\subsection{Experimental procedures and parameters}

The monochromatic photon beam from the BW3 beamline at DORIS III passed into the experimental chamber so that the interaction region of the I-TOF was placed at the focus of the beamline. We set a constant extractor field in our interaction region and used the positron bunch signal as the trigger to start taking measurements, with an event recorded when the first ionized atom hit the electron multiplier after the bunch trigger, giving us the flight time of the ion. The operation mode of DORIS III circulates only five positron bunches between 192 and $196 \mathrm{~ns}$ apart. Due to this short time between the bunches, the slower ions did not have enough time to move all the way down the drift tube before the next positron bunch triggered a reaction, and their signal peaks could overlap with the signal peaks of faster ions. However, by setting the extractor field to high enough values and adjusting the drift tube potential accordingly, we could accelerate all ions so that their signal peaks did not overlap in the spectrum. We could then identify the separated signal peaks and get the ratios we needed to evaluate the performance of the spectrometer.

The Ne gas used in the experiment was of $99.9 \%$ purity or better. The base chamber pressure before the gas was let in was in the $\times 10^{-8}$ mbar range. The pressure in the chamber while 


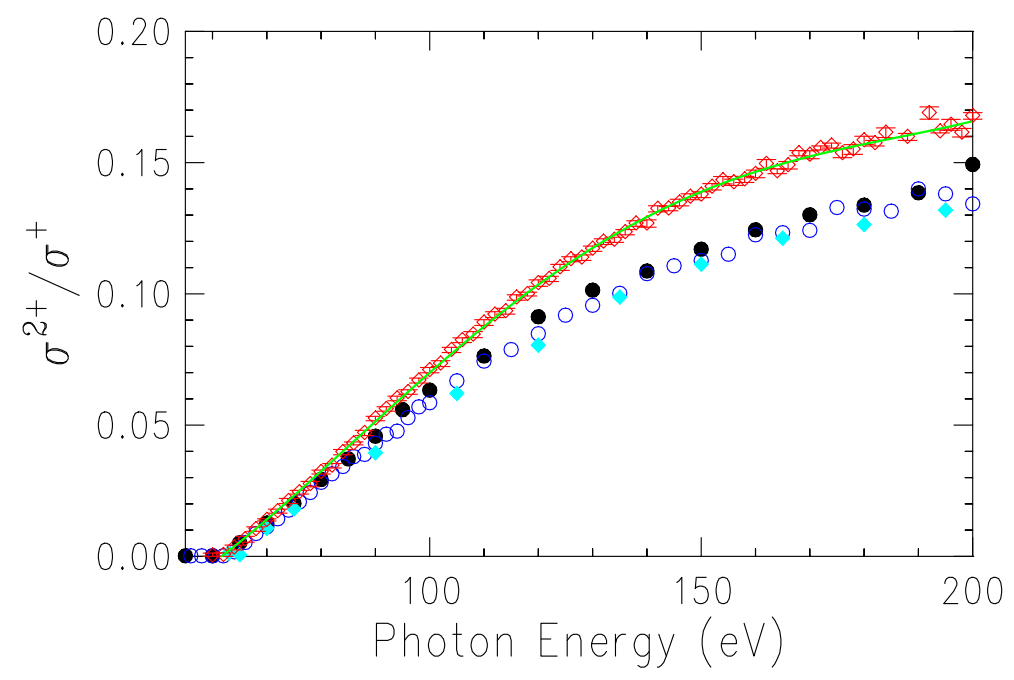

Figure 3. (Color online) $\mathrm{Ne} \sigma^{2+} / \sigma^{+}$taken from the data from the BW3 beamline compared with the reference data. The red open diamonds with error bars are the data from BW3, the blue open circles are from [7], the filled black circles are from [8], and the azure filled diamonds are from [9]. The thick green line is the polynomial fit to the data we took at BW3.

performing the measurements was in the order of $10^{-7}$ mbar, and we estimated the pressure in the interaction region being around $10^{-5}$ mbar due to the focusing effect of the needle gas inlet system. Energy scans of the gas target were taken from the double photoionization threshold up to $200 \mathrm{eV}$ in $2 \mathrm{eV}$ steps at 100 seconds per spectrum. The photon energy calibration was performed using the $\mathrm{Xe} 4 d \rightarrow 6 p_{3 / 2}$ resonance [16]. The resulting deviations from literature values were on the order of the bandwith and were therefore not corrected.

\section{Data analysis}

The analysis of the data consisted of two steps. We first took the energy scans of Ne, evaluating the gas' double-to-single photoionization cross section ratio from the double-photoionization thresholds to $200 \mathrm{eV}$, and then modeled the curve to extract a function. Secondly, we evaluated the slope of the model curve and the error bars of our ratio measurement to estimate the measurement error for the photon energy. Because we took data only up to $200 \mathrm{eV}$, we did not have triply photoionized $\mathrm{Ne}$ over the range, and we had no inner shell effects-all of the excited electrons were in neon's valence shell.

In figure 3 we compared the Ne curve against the curves found in the literature [7-9]. We fitted our neon curve with a simple fourth-order polynomial fit. The fit curve is also shown in figure 3. As one can see, the curve derived from the data taken at the BW3 beamline generally matches the data from other sources, though our ratio is about $10 \%$ higher than that the literature values. The difference could lie in a too-low voltage on the electron multiplier that would have over-estimated the higher charged states. We could not use a higher electron multiplier potential as we were already applying the recommended maximum voltage of the electron multiplier.

To gauge the usefulness of the proposed calibration principle, we had to convert the error bars for the double-to-single photoionization ratio to error bars for the photon energy, which is the 


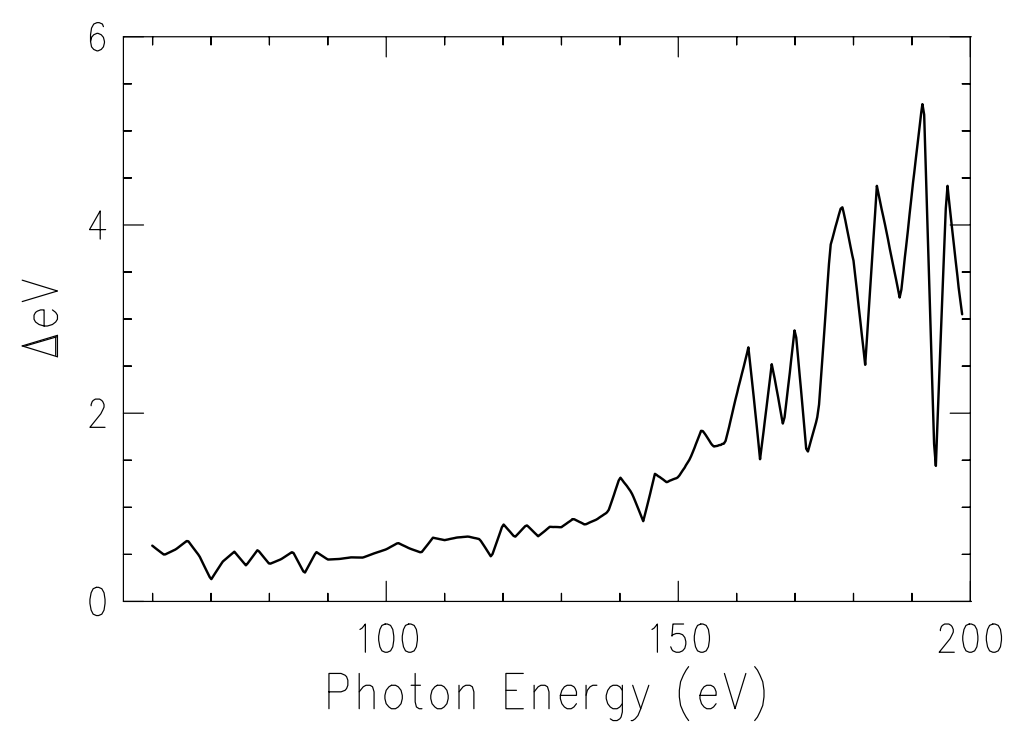

Figure 4. (Color online) The uncertainty $\Delta \mathrm{eV}$ for the measurement of the photon energy of neon from 59 to $200 \mathrm{eV}$ based on the experimental data at BW3.

quantity to be derived. To do so, we simply divided the $\Delta\left(\sigma^{2+} / \sigma^{+}\right)$- the uncertainty in our ratio measurement for a data point - with the slope of the polynomial fit curve at that point. This gave us a $\Delta \mathrm{eV}$, the uncertainty in photon energy at this particular $\sigma^{2+} / \sigma^{+}$value. Figure 4 shows the $\Delta \mathrm{eV}$ at every photon energy we scanned.

\section{Results and discussion}

As figure 4 shows, the uncertainty in the photon energy measurement $\Delta \mathrm{eV}$ is very low near the $60 \mathrm{eV}$ neon double photoionization threshold, being in the range of $\Delta \mathrm{eV}=0.4-0.6 \mathrm{eV}$, corresponding to a $1 \%$ uncertainty or better. The uncertainty stays below about $0.6 \mathrm{eV}$ up to about $115 \mathrm{eV}$, where it begins to rise. The uncertainty stays at or below $1 \%$ below $150 \mathrm{eV}$. This result is expected since the slope of the double-to-single photoionization ratio of neon is steep in this region until it becomes flatter in the $150-200 \mathrm{eV}$ range, as seen in figure 3. At these higher photon energies, the uncertainty in the ratio can be transformed into a larger uncertainty in the photon energy, as the values of the ratios at the higher energies are closer to each other than at the lower energies, where the slope of the curve is steeper. Despite this effect, the accuracy of the measurements would be good enough to detect a photon energy drift or fluctuations at FLASH. At photon energies above $150 \mathrm{eV}$, other rare gases with different photoionization ratio curves could be used to improve the calibration method.

\section{Conclusions}

We have performed the first test of the ion time-of-flight online photoionization spectrometer meant for FELs. The test showed that the I-TOF spectrometer is a viable idea that can be used at an FEL to measure the photon energy of the beam without significant interference with the light and therefore 
it will make a good addition as a reliable and simple tool at FLASH for online photon diagnostics. Though more work needs to be done to modify the spectrometer to take data at FLASH, and though the data taking and analysis need to be automatized, these tasks are fairly standard. With further tests with other gases that could cover other energy ranges, we will be able to accurately cover the whole energy range of FLASH as well as future FELs.

\section{References}

[1] J. Pflüger, Undulators for SASE FEL's, Nucl. Instrum. Meth. A 445 (2000) 366.

[2] V. Ayvazyan et al., First operation of a free-electron laser generating $G W$ power radiation at $32 \mathrm{~nm}$ wavelength, Eur. Phys. J. D 37 (2006) 297.

[3] W. Ackermann et al., Operation of a free-electron laser from the exterme ultraviolet to the weater window, Nat. Photonics 1 (2007) 336.

[4] K. Tiedtke et al., The soft x-ray free-electron laser FLASH at DESY: beamlines, diagnostics and end-stations, New J. Phys. 11 (2009) 023029.

[5] M. Wellhöfer et al., Photoelectron spectroscopy as a non-invasive method to monitor the SASE-FEL spectra, 2008 JINST 3 P02003.

[6] K. Tiedtke et al., Gas detectors for x-ray lasers, J. Appl. Phys. 103 (2008) 094511.

[7] J.M. Bizau and F.J. Wuilleumier, Redetermination of absolute partial photoionization cross section of He and Ne atoms between 20 and $300 \mathrm{eV}$ photon energy, J. Electron Spectrosc. 71 (1995) 205.

[8] I.H. Suzuki and N. Saito, $\gamma$-value in rare gases for soft $x$-ray absolute maeasurement, ETL Tech. Rep. 56 (1992) 687.

[9] R.J. Bartlett et al., Single-photon double ionization of He and Ne, Phys. Rev. A 46 (1992) 5574.

[10] T.N. Chang and R.T. Poe, Double photoionizaton of neon, Phys. Rev. A 12 (1975) 1432.

[11] S.L. Carter and H.P. Kelly, Double photoionizaton of neon and argon, Phys. Rev. A 16 (1977) 1525.

[12] W.C. Wiley and I.H. McLaren, Time-of-flight mass spectrometer with improved resolution, Rev. Sci. Instrum. 26 (1955) 1150.

[13] C.U.S. Larsson et al., First results from the high resolution XUV undulator beamline BW3 at HASYLAB, Nucl. Instrum. Meth. A 337 (1994) 603.

[14] R. Reininger and V. Saile, A soft x-ray grating monochromator for undulator radiation, Nucl. Instrum. Meth. A 288 (1990) 343.

[15] A.R.B. de Castro and R. Reininger, Optimization of undulators for a SX-700 instrument: finite element coupled to ray tracing, Rev. Sci. Instrum. 63 (1992) 1317.

[16] G.C. King, M. Tronc, F.H. Read and R.C. Bradford, An investigation of the structure near the $L_{2,3}$ edges of argon, the $M_{4,5}$ edges of krypton and the $N_{4,5}$ edges of xenon, using electron impact with high resolution, J. Phys. B 10 (1977) 2479. 